\title{
Chemotherapy use and quality of life in cancer patients at the end of life: an integrative review
}

\author{
Elham Akhlaghi ${ }^{1,2}$, Rebecca H. Lehto ${ }^{1,2}$, Mohsen Torabikhah ${ }^{3}$, Hamid Sharif Nia ${ }^{4 *}$ (D) Ahmad Taheri ${ }^{3}$, \\ Ehsan Zaboli ${ }^{5}$ and Ameneh Yaghoobzadeh ${ }^{6}$
}

\begin{abstract}
Background: When curative treatments are no longer available for cancer patients, the aim of treatment is palliative. The emphasis of palliative care is on optimizing quality of life and provided support for patients nearing end of life. However, chemotherapy is often offered as a palliative therapy for patients with advanced cancer nearing death. The purpose of this review was to evaluate the state of the science relative to use of palliative chemotherapy and maintenance of quality of life in patients with advanced cancer who were at end of life.
\end{abstract}

Materials and methods: Published research from January 2010 to December 2019 was reviewed using PRISMA guidelines using PubMed, Proquest, ISI web of science, Science Direct, and Scopus databases. MeSH keywords including quality of life, health related quality of life, cancer chemotherapy, drug therapy, end of life care, palliative care, palliative therapy, and palliative treatment.

Findings: 13 studies were evaluated based on inclusion criteria. Most of these studies identified that reduced quality of life was associated with receipt of palliative chemotherapy in patients with advanced cancer at the end of life.

Conclusion: Studies have primarily been conducted in European and American countries. Cultural background of patients may impact quality of life at end of life. More research is needed in developing countries including Mideastern and Asian countries.

Keywords: Quality of life (QOL), Health related quality of life (HRQOL), Cancer chemotherapy, Drug therapy, End of life care, Palliative care, Palliative therapy, Palliative treatment

\section{Introduction}

Best practices for management of advanced cancer are of global health concern, particularly in developing countries [1]. When curative strategies are exhausted, the focus of cancer care shifts to maintenance of quality of life (QOL) and extension of survival [2]. Studies indicate that -20 to $50 \%$ of patients with advanced cancers receive chemotherapy $(\mathrm{CT})$ towards end of life with the

*Correspondence: h.sharifnia@mazums.ac.ir; pegadis@yahoo.com

${ }^{4}$ Department of Nursing, Mazandaran University of Medical Science, Sari, Iran

Full list of author information is available at the end of the article aims of extending survival and improving QOL [3-5]. Such rigorous treatment strategies may contribute to death in non-preferred environments such as the intensive care unit (ICU), incurring additional costs for care that is often futile and that may also diminish QOL in the patients' final days. Intensive medical management at end of life may also limit the opportunity for patients to receive hospice services, supportive care that focuses on enhancing comfort and promoting quality of life when facing death [6]. The purpose of the review was to examine the state of the science relative to the use of $\mathrm{CT}$ in patients with advanced cancers at the end of life, and to original author(s) and the source, provide a link to the Creative Commons licence, and indicate if changes were made. The images or other third party material in this article are included in the article's Creative Commons licence, unless indicated otherwise in a credit line to the material. If material is not included in the article's Creative Commons licence and your intended use is not permitted by statutory regulation or exceeds the permitted use, you will need to obtain permission directly from the copyright holder. To view a copy of this licence, visit http://creativecommons.org/licenses/by/4.0/. The Creative Commons Public Domain Dedication waiver (http://creativeco mmons.org/publicdomain/zero/1.0/) applies to the data made available in this article, unless otherwise stated in a credit line to the data. 
evaluate the impact of such treatments on patients QOL as they near death.

Chemotherapy generally refers to treatments aimed at stopping or eradicating the growth of cancer cells that are administered orally, intravenously, intrathecally, by injection, or subcutaneously depending on the type and stage of cancer being treated [7]. For patients with advanced cancers, many are now receiving oral antineoplastic agents [8]. Chemotherapies (CT) in general are associated with a host of side effects and symptoms that are specific to the type of agent and individualized patient characteristics. Numerous studies have identified the impact of CT on QOL, with some research suggesting that patients may experience improved QOL and length of survival when offered such treatments as a last alternative [9]. However, CT are also associated with increasing the cost of care at end of life due to the additional costs associated with need for pharmaceuticals, potential for blood products, laboratory and diagnostic testing, health professional support and unscheduled visits that result in hospitalization $[10,11]$.

Palliative care is aimed at providing comprehensive support and comfort via a multidisciplinary team approach to enhance QOL when cure is not an option [12]. Some research has shown that stoppage of intensive medical treatments and providing palliative care have improved both QOL and length of survival of advanced cancer patients [13]. Further, the incorporation of early specialized palliative care may reduce the risk of receiving CT during the final two weeks of life [14]. Many patients who have good performance status, however, receive palliative $\mathrm{CT}$ as they near end of life with the aim of increasing survival length and even to improve QOL [10].

According to the Health Service Research Committee of the American Society of Clinical Oncology (ASCO), CT can potentially improve QOL in late stages of life even if it doesn't impact survival length [13]. Further, medical decision making relative to use of CT in patients nearing the end of life may be fostered by the development and availability of newer anticancer agents that have fewer side effects [15]. However, ASCO and the National Quality Forum have identified that other factors impact QOL at end of life (defined as final 30 days of life) and should be considered, such as unscheduled emergency room visits, the potential for lengthy hospitalizations, ICU admissions, and ICU deaths [16]. Recently, ASCO contended that stopping $\mathrm{CT}$ in cancer patients at the end of life is one of five factors which can improve the quality of patient care and reduce healthcare costs [16]. There remain limited studies that have systematically evaluated the effects of palliative CT on the QOL of cancer patients nearing end of life [4, 16, 17]. Some studies evaluate factors such as age, sex, type of cancer, and health care system characteristics while omitting information about perceived QOL [14]. Research findings suggest that culture may impact the utilization of aggressive treatment in patients with advanced cancer. For example, a study from Japan stated that only $3.7 \%$ of patients receive $\mathrm{CT}$ in their last 2 weeks of life [18]. In spite of increasing use of CT at end of life in recent years, the impact on patients' QOL remains equivocal. Therefore, the purpose of this review was to evaluate what is known about the use of $\mathrm{CT}$ and associated QOL in patients with advanced cancer approaching end of life.

\section{Methods}

\section{Research question}

The research question aimed to examine: what is the state of the science between use of palliative CT and QOL in patients with advanced cancer at end of life?

\section{Search strategy}

An integrative review was conducted in 2019. Studies published between January 2010 and December 2019 were reviewed using PRISMA guidelines (see Fig. 1). Six databases (PubMed, Proquest, ISI web of science, Science Direct, Scopus, SID) were searched using AND and OR Boolean operators. MeSH keywords including quality of life (QOL), health related quality of life (HRQOL), cancer chemotherapy, drug therapy, end of life care, palliative care, palliative therapy, and palliative treatment were used to search for associated studies.

\section{Eligibility criteria}

Inclusion criteria were: (1) Persian and English articles published between January 2010 to December 2019 that were freely accessible; (2) patients with advanced cancer at the end of life; (3) evaluation of anti-cancer CT and patients' QOL; and (4) quantitative data based (including retrospective, prospective cohort, cross-sectional studies,). Exclusion criteria were studies that evaluated specific CT regimens without an inquiry of patients' QOL, and use of other treatment modalities in conjunction with CT. Also eliminated were interventional studies that did not address the relationships between use of palliative CT and QOL in patients with advanced cancer nearing end of life. The Boolean operators like OR (for synonymous keywords) and AND (for relating different keywords) was performed to search the articles. Utilizing these established inclusion and exclusion criteria, abstracts of retrieved articles were first reviewed and those that matched the criteria were evaluated. After duplicates were removed, two researchers independently reviewed the included articles and concurrence was achieved with 13 papers finally selected. The search 


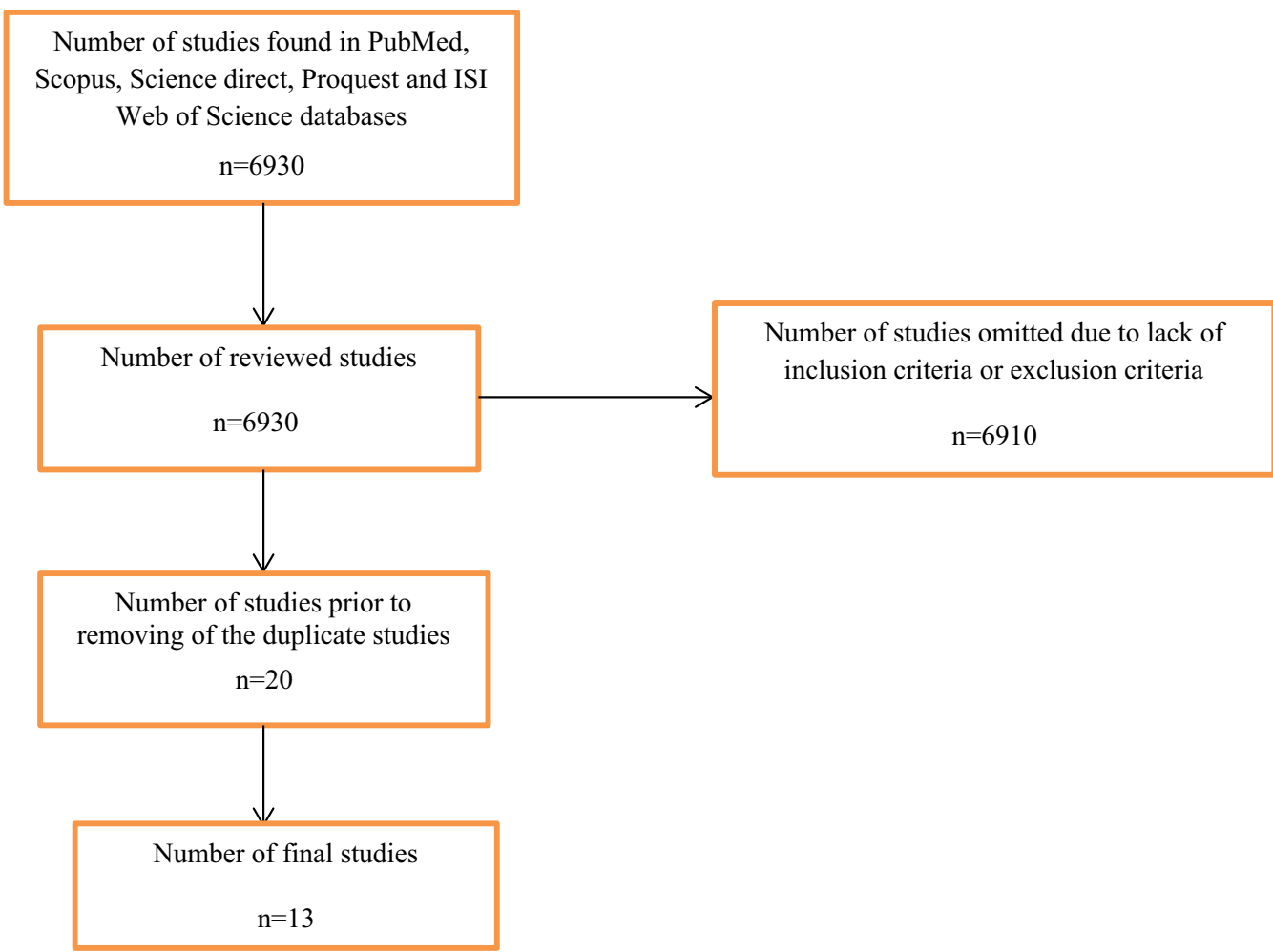

Fig. 1 Systematic review search based on PRISMA

Table 1 Number of articles found from respective databases

\begin{tabular}{lcc}
\hline Databases & Number of articles & $\begin{array}{l}\text { Selected } \\
\text { articles }\end{array}$ \\
\hline PubMed & 5627 & 7 \\
Proquest & 960 & 6 \\
ISI web of Science & 166 & 5 \\
Science Direct & 90 & 0 \\
Scopus & 87 & 2 \\
SID & 0 & 0 \\
Total & 6930 & 20 \\
\hline
\end{tabular}

results are presented in Table 1 . The steps for choosing articles based on PRISMA guidelines are also shown in Fig. 1.

\section{Quality assessment (risk of bias)}

The quality of the studies was separately measured by two researchers using two tools, the Quality Assessment of Diagnostic Accuracy Studies (QUADAS-2) and the checklist of Standards for Reporting of Diagnostic Accuracy (STARD) [19]. These tools examine the risk bias in accuracy and comprehensiveness of study reports. The combination of both tools, with revision of some cases, improved the examination of the included studies [20]. QUADAS-2 was used to measure the internal stability of the studies studied. The tool consists of items with threeoption answers (i.e., yes, no, and unknown). A 25-item STARD tool was used to evaluate the study design quality (including sample collection and data). The combination of these two tools and their associated flexibility helped make them compatible with the study goals [20]. Standardized methods were used to ensure the quality assessment and to ensure the inter-rater reliability of both analyses. Finally, findings from the two assessments were evaluated to ensure that they were comparable [21].

\section{Results}

Of the 13 studies, five studies were conducted in the United States (U.S.), six were from Europe, and one were from Australia and India respectively. Based on studies that reported this information, the sampling interval across the 13 studies were from 2002 to 2012 (Table 2).

\section{General characteristics of the included studies}

The included studies incorporated patients with advanced cancers who had undergone palliative CT. Sample sizes ranged from 40 [22] to 2313 [23] participants 


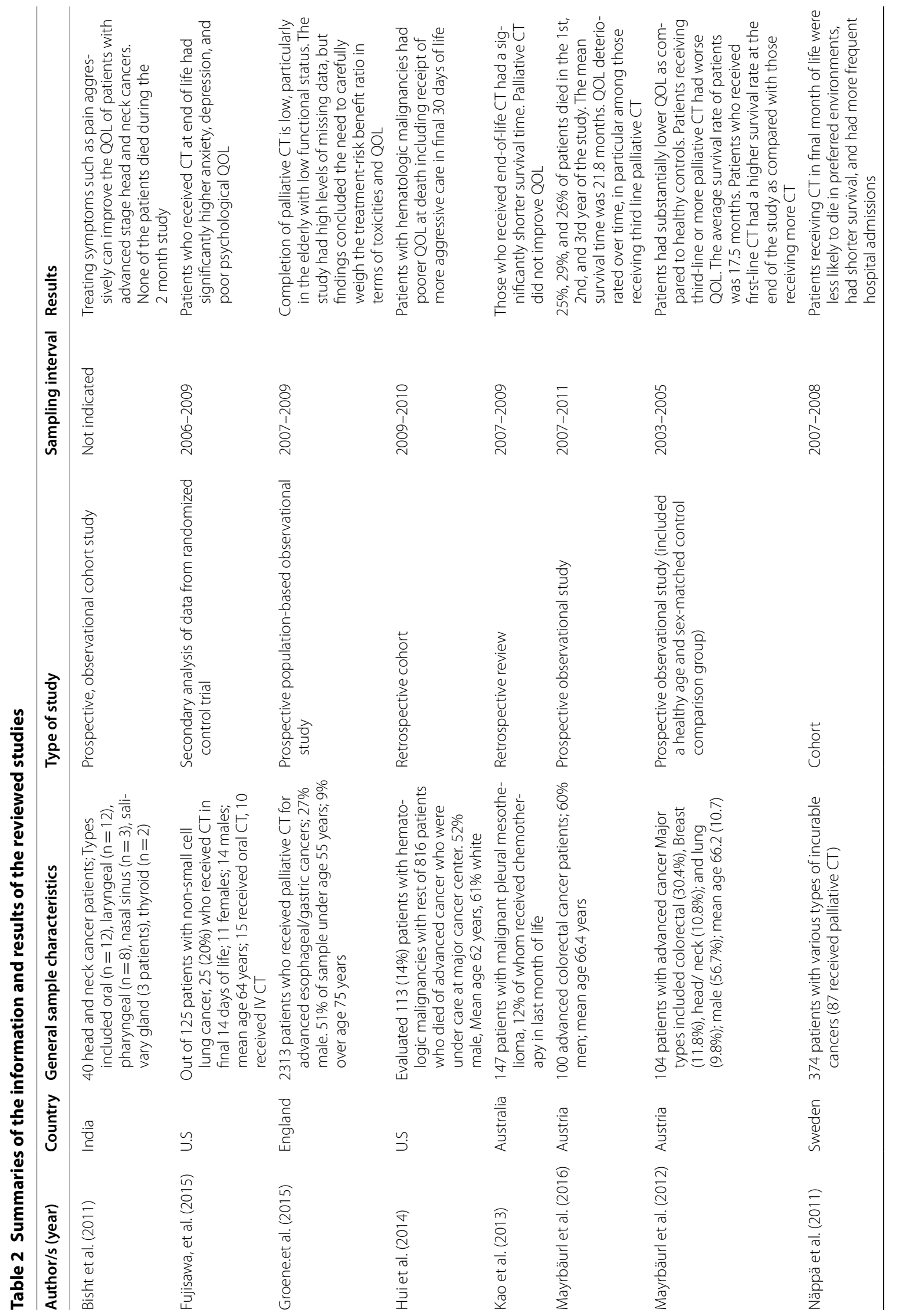




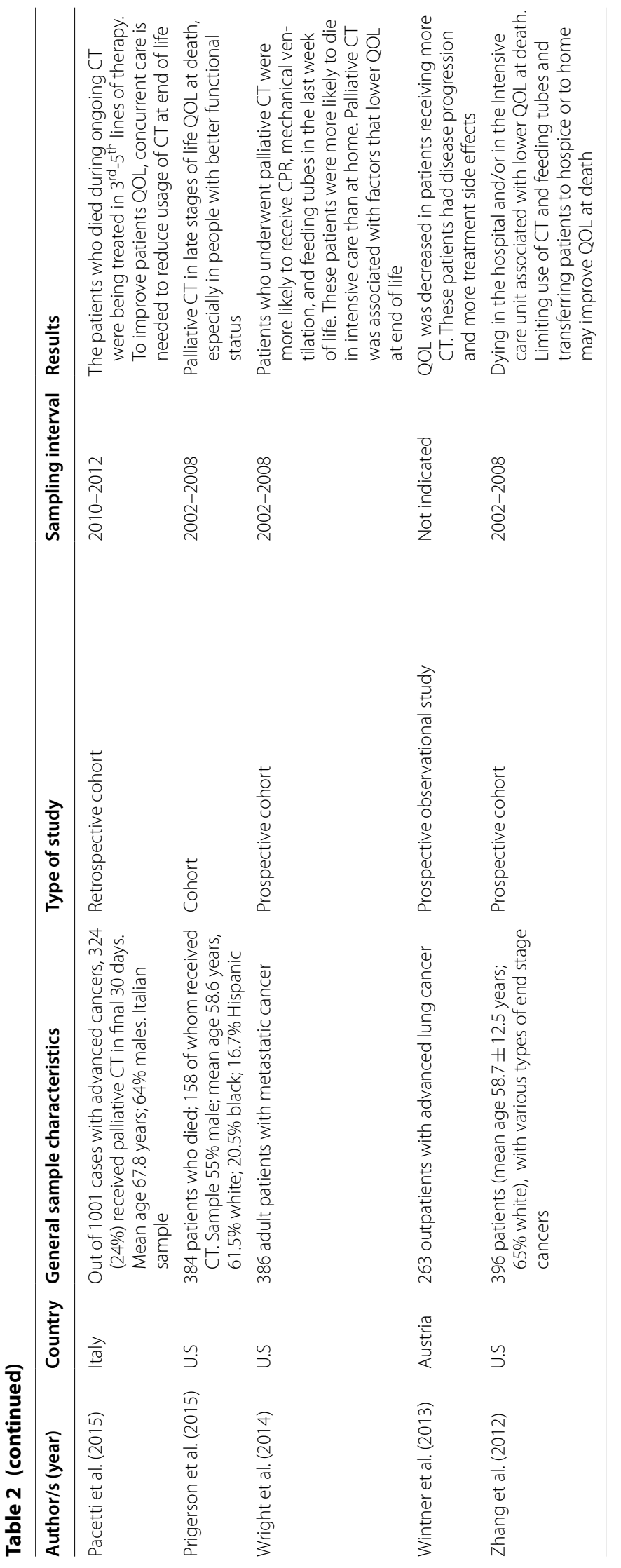


with an age range between 50 and 70 years old. Most study participants were male (see Table 3 for demographic characteristics). Quality of life was measured primarily with questionnaires including the Quality of Life Questionnaire-C30 (EORTC QLQ-C30), an internationally validated reliable instrument that evaluates $\mathrm{QOL}$ in social, role, cognitive, emotional, and physical functioning domains $[16,18,24]$. The European Cooperative Oncology Group (ECOG) performance status [3, 4, 16], the McGill Quality of Life Questionnaire [25], and Padilla's Quality of Life Index [14] were also used. Other factors use to evaluate QOL included place of death and use of aggressive therapies at end of life $[3,12]$.

\section{Main outcomes}

Prigerson et al. (2015) conducted a prospective, multiinstitution cohort study of patients with end-stage solid tumor cancers from different cancer centers across the U.S. to determine the relationships between use of CT and QOL as patients neared death. Out of 660 patients with late stage cancer, 384 died during the study period and these patients were evaluated as they approached death. Of these patients, 158 (about half, were receiving $\mathrm{CT}$ at study enrollment (4 months median time before death). The patients who received CT were significantly younger, better educated, had few comorbid conditions, had better performance status and were more likely to receive treatment at academic cancer centers. Further, they were more likely to have either breast or pancreatic cancer diagnoses. Findings demonstrated that patients with better performance status had a worse quality of death even when controlling for intensive care at end of life. For those patients with low to moderate performance status, CT use was unrelated to quality of death. The researcher's findings indicated that CT use at end of life had no benefits to length of survival, and impaired QOL in the patients' final days [4].

A secondary analysis of data from a randomized clinical trial evaluating palliative care for patients with advanced non-small cell lung cancer were analyzed to determine predictors of use of CT in the final two weeks of life. Study findings demonstrated that of 125 patients who died, $20 \%(n=25)$ had received $C T$ at end of life. Of these 25 patients, 15 were receiving oral CT whereas 10 were receiving intravenous $\mathrm{CT}$ or a combination of both [25]. Patients who received CT at end of life had worse psychological health including significantly more anxiety, depressive symptoms, and poorer QOL [25].

Hui and colleagues conducted a retrospective cohort study of patients with advanced cancer who died while receiving care at a major U.S. Cancer Center. They conducted a comparative analysis of care for 113 patients with hematologic malignancies (14\% of sample) with patients who had solid tumor cancers $(n=816)$ [16]. The patients who died with hematologic malignancies were significantly more likely to receive aggressive treatments in their final 30 days compared to the group of patients with solid tumor cancers. Such care included ICU admissions, CT and targeted therapies, prolonged hospitalizations, and emergency unscheduled use of services [16] that culminated in lower QOL at death.

Table 3 Demographic characteristics of the studied units in the selected studies

\begin{tabular}{|c|c|c|c|c|c|c|}
\hline \multicolumn{2}{|l|}{ Sex (male) } & \multicolumn{2}{|l|}{ Age } & \multirow{2}{*}{$\begin{array}{l}\text { Number } \\
\text { of chemotherapy } \\
\text { samples }\end{array}$} & \multirow{2}{*}{$\begin{array}{l}\text { Total number } \\
\text { of samples }\end{array}$} & \multirow[t]{2}{*}{ Study } \\
\hline $\begin{array}{l}\text { The number of recipients } \\
\text { of palliative chemotherapy } \\
\text { (male) }\end{array}$ & $\begin{array}{l}\text { Total } \\
\text { Number } \\
\text { (male) }\end{array}$ & $\begin{array}{l}\text { Medium age } \\
\text { recipients of palliative } \\
\text { chemotherapy }\end{array}$ & Average age & & & \\
\hline$-^{a}$ & 28 & $-{ }^{a}$ & 55 & 24 & 40 & Bisht \\
\hline 63 & $-{ }^{a}$ & 64.5 & 64 & 125 & 151 & Fujisawa \\
\hline 1717 & 6339 & 55 & $-{ }^{a}$ & 2313 & 9768 & Groene \\
\hline$-^{a}$ & 182 & $-^{a}$ & 62 & 350 & 816 & Hui \\
\hline 7 & 135 & $-{ }^{a}$ & 73 & 9 & 147 & Kao \\
\hline 60 & 60 & 66.4 & 66.4 & 100 & 100 & Mayrbäurl 2016 \\
\hline 59 & 59 & 66.2 & 66.2 & 104 & 104 & Mayrbäurl 2011 \\
\hline 49 & 190 & 65 & 65.5 & 87 & 374 & Napa \\
\hline 105 & 105 & 67.8 & 67.8 & 162 & 162 & Pacetti \\
\hline 85 & 171 & 56.3 & 58.6 & 158 & 312 & Prigerson \\
\hline 119 & 215 & 56.4 & 58.4 & 216 & 386 & Wright \\
\hline 112 & 112 & 67.1 & 67.1 & 187 & 187 & Wintner \\
\hline 216 & 389 & $-{ }^{a}$ & $-{ }^{a}$ & 396 & 396 & Zhang \\
\hline
\end{tabular}

a Information not available 
In a retrospective cohort study evaluating patients with advanced metastatic cancer during their last month of life, Pacetti et al. (2015) found that 162 patients out of 2164 at a major Italian Oncology Center received CT prior to death. Of these patients who received CT in their final 30 days, about $65 \%$ were males, and the individual health provider was the only predictor for why CT was continued at end of life [13].

Wright et al. (2014) conducted a secondary analysis of data from a large federally funded prospective, longitudinal, multi-institutional study in the U.S. of terminally ill patients with advanced cancer. Data from 386 patients who died during the study were analyzed to determine whether palliative CT administration in the final months of life was related to intensity of medical care and location of death [3]. Patients who received palliative CT (56\% of the sample) as compared with those who did not receive palliative $\mathrm{CT}$ were significantly more likely to die in a non-preferred environments, were more likely to be hospitalized in an ICU at time of death, and were also more likely to receive cardiopulmonary resuscitation and/or ventilator support [3].

In a retrospective review study, Kao et al. (2013) evaluated factors associated with use of CT at the end of life in Australian patients with malignant mesothelioma. Of 147 patients who died, 21 received treatment in the last month of life [26]. For those patients receiving CT during the last month of life, the only factor associated with its' use was having at least two previous cycles of treatment. These patients who received CT had significantly shorter survival as compared to patients who did not receive $\mathrm{CT}$ and there was a trend towards death outside the home. A limitation of the study was a lack of information about where these deaths occurred. Importantly the study suggests that end of life planning is less likely to occur when patients continue with $\mathrm{CT}$ in the terminal phase of life [26].

In a study from Sweden, Nappa and his colleagues (2011) evaluated characteristics associated with use of palliative $\mathrm{CT}$ in patients with advanced stage epithelial cancers in the last month of life. Of 374 patients, 87 (23\%) received palliative CT in the last month of life [24]. Use of palliative CT was associated with shorter survival, increased hospital admissions, and less likelihood of dying at home. Further, there was less documented evidence of discussion about ceasing treatment with patients who received palliative $\mathrm{CT}$ during the last month of life.

In a large federally funded longitudinal prospective cohort study in the U.S., factors associated with QOL at end of life were evaluated in 396 patients with advanced cancer and their caregivers. Factors that were most associated with lower QOL at end of life included death in intensive care and receipt of life-prolonging therapies [2]. Better patient QOL was associated with positive relationships with their physician and pastoral spiritual care provision [2]. Other factors that improved QOL at death included personal religious activities, caregiver health, and better perceived mental health [2].

In another prospective cohort study, Bisht et al. (2011) evaluated 40 patients with advanced stage head and neck cancer who were receiving palliative care. All patients had stage 4 cancer and had recurrence and metastasis to distant areas. Of the 40 patients, 24 received palliative CT, in conjunction with other medications such as analgesics, steroids, and anti-emetics for symptom management. Findings identified that QOL was positively impacted by pharmacologic management of symptoms [22].

Mayrbaurl et al. (2016) conducted a prospective observational study that evaluated the QOL of 100 patients with advanced colorectal cancer who received first, second, or third lines of palliative chemotherapy and were followed for up to 3 years [27]. At the onset of the study, 73 patients began first-line of palliative CT, and 27 were at the start of second-line palliative CT. Ongoing QOL assessment was continued until patients' were unable to complete the questionnaires, death occurred, or the conclusion of the 3 year study was completed [27]. Quality of life progressively declined with the continuation of palliative CT and disease advancement [27]. Mayrbäurl et al. (2012) also conducted a prospective observational study at an Austrian health center that compared QOL in patients with mixed advanced stage cancer diagnoses preparing for palliative CT to healthy age and sexmatched controls. Quality of life of patients was assessed at baseline and during the palliative CT [28] with similar comparative lower QOL identified.

Groene and colleagues conducted a prospective population-based observational study of factors associated with completion of palliative CT in patients with advanced esophageal and gastric cancers in England. There were 2313 (about 24\% of total) patients who received palliative $\mathrm{CT}$ [23]. Where data were available, only 50-60\% completed treatment among those with good performance status [16]. Findings conclude low likelihood of treatment completion (53\% overall).

Wintner et al. (2013) evaluated QOL in patients with advanced lung cancer who were receiving varying outpatient palliative CT treatment lines [29]. Findings showed that QOL remained essentially unchanged but was worse in those receiving advanced lines of CT [18].

\section{Discussion}

Given progress in anti-cancer CT strategies for patients with advanced cancers, there is an increasing use of palliative CT at the end of life [15]. 
The purpose of this study was to evaluate relationships between use of palliative CT and QOL in patients with advanced cancer nearing end of life. Generally, the results of most of the selected studies indicated that palliative CT was associated with reduced QOL for patients. For example, such therapy was associated with increased necessity for cardiopulmonary resuscitation, mechanical ventilation, use of a feeding tube, and death in intensive care [3]. Further, such treatment was also associated with shortened survival and reduced quality of death [4].

Some patients with advanced cancer may prefer to receive aggressive treatment even if the therapies are associated with toxicities and side effects [30]. A study by Kao et al. found that patients who received palliative CT had shorter survival time and more frequent hospital admissions including unscheduled emergency department visits compared to those who did not receive $\mathrm{CT}$ at the end of life [26]. The goal of palliative CT is to maintain or improve QOL, improve length of survival and reduce symptoms in oncologic emergency conditions [1]. The usefulness of CT should always be considered by associated toxicities and side effects, given potential impact on QOL [1]. The presence of adverse effects from $\mathrm{CT}$ in cancer patients at the end of life was noted in the assessed studies $[4,9]$.

Early provision of palliative care with a multidisciplinary team has offered multiple benefits for patients with advanced cancer. For example, Temel and her colleagues compared two groups of patients who were newly diagnosed with metastatic cancer; one group that received palliative care with standard oncological care and the other group who received only standard oncology care. Findings demonstrated that the group that received integrated early palliative care had comparatively significant improvement in QOL, mood, and length of survival [12]. A complex issue is the use of palliative $\mathrm{CT}$ as a supportive strategy to improve length of survival, bolster QOL, assist with symptom management, and reduce disease progression given it may also result in more aggressive care at end of life [8]. Regimens and types of CT used in the palliative context vary. Further, it is often not clear that patients' who receive these treatments have clearly addressed their end of life wishes and have advanced directives in place. Patients may die while receiving anti-cancer regimens because of death denial and/or communication barriers with their oncology team. For example, it may be more challenging to discuss stoppage of treatment as opposed to recommending further CT for patients who are nearing end of life [8] especially when patients are younger and have reasonable performance status. The mental health of patients at end of life and communication with their health providers may also impact the type of decisions patients will make in regards to sustaining treatment [31]. Without clear guidelines, the continued problems with medically futile treatments and untoward deaths in highly costly and non-preferred environments are likely to persist.

The review has limitations. These limitations include the possibility that articles that pertain to use of CT at end of life in patients with cancer were missed. For example, some studies may not include oral CT when evaluating use of CT at end of life [18]. Further, given the complex issues associated with patients who have advanced cancers, e.g., age, developmental stage, type of disease, comorbid conditions, etc., it can be challenging to determine best approaches to optimize individualized care at end of life. Cultural factors including the role of family caregivers may impact whether or not palliative $\mathrm{CT}$ is offered and utilized, and whether or not death occurs in a preferred environment. More research is needed that examines such issues across cultures, especially as more types of CT are made available to patients with advanced cancers. Research that includes diverse samples from global samples remains needed. Many of the current studies were conducted at major tertiary cancer centers so may not be reflective of practices in nonurban communities. Another limitation relates to the lag in sampling intervals among the published papers (20022012). Studies with more recent data collection could better reflect current practices.

\section{Conclusions}

The results of most selected studies show that palliative $\mathrm{CT}$ is associated with a decrease in QOL for patients. However, studies have been conducted primarily in European and American countries, and patients' cultural backgrounds may affect quality of life at the end of life. More research is needed in developing countries, including the Middle East and Asia.

\section{Abbreviations \\ QOL: Quality of life; CT: Chemotherapy; ICU: Intensive care unit; ASCO: Ameri- can Society of Clinical Oncology; HRQOL: Health related quality of life; QUA- DAS: Quality Assessment of Diagnostic Accuracy Studies; STARD: Standards for Reporting of Diagnostic Accuracy; U.S.: United States; QLQ-C30: Quality of Life Questionnaire-C30; ECOG: European Cooperative Oncology Group.}

\section{Acknowledgements}

Thanks to everyone who helped us with this research.

\section{Authors' contributions}

Conceived and designed the study; HSH, EA, RL and MT. Collecting data; HSH, EA, RL, MT, AT, EZ and AY. Analyzed data; HSH, EA, RL and MT. Write manuscripts; HSH, EA, RL and MT. All authors participated in the development of this manuscript. All authors read and approved the final manuscript.

Funding

This work was supported by Mazandaran University of Medical Sciences. 


\section{Ethics approval and consent to participate}

The protocol of this study was approved by the Mazandaran University of Medical Sciences Research Ethics Committee (IR.MAZUMS.REC.1398.5938),

\section{Consent for publication}

Not applicable.

\section{Availability of data and materials}

Not applicable.

\section{Competing interests}

Not applicable.

\section{Author details}

${ }^{1}$ School of Nursing and Midwifery, Iran University of Medical Sciences, Tehran, Iran. ${ }^{2}$ College of Nursing, Michigan State University, East Lansing, MI, USA. ${ }^{3}$ Department of Adult Health Nursing, Nursing and Midwifery Faculty, Isfahan University of Medical Sciences, Isfahan, Iran. ${ }^{4}$ Department of Nursing, Mazandaran University of Medical Science, Sari, Iran. ${ }^{5}$ Gastrointestinal Cancer Research Center, Mazandaran University of Medical Sciences, 48166-33131 Sari, Iran. ${ }^{6}$ Tehran University of Medical Sciences, Tehran, Iran.

Received: 10 April 2020 Accepted: 24 September 2020

Published online: 07 October 2020

\section{References}

1. Urruticoechea A, Alemany R, Balart J, Villanueva A, Vinals F, Capella G. Recent advances in cancer therapy: an overview. Curr Pharm Des. 2010;16(1):3-10.

2. Zhang B, Nilsson ME, Prigerson HG. Factors important to patients' quality of life at the end of life. Arch Intern Med. 2012;172(15):1133-42.

3. Wright AA, Zhang B, Keating NL, Weeks JC, Prigerson HG. Associations between palliative chemotherapy and adult cancer patients' end of life care and place of death: prospective cohort study. BMJ (Clin Res Ed). 2014;348:g1219.

4. Prigerson HG, Bao Y, Shah MA, Paulk ME, LeBlanc TW, Schneider BJ, et al. Chemotherapy use, performance status, and quality of life at the end of life. JAMA Oncol. 2015;1(6):778-84.

5. Karver SB, Berger J. Advanced care planning-empowering patients for a peaceful death. Asian Pac J Cancer Prev APJCP. 2010;11(Suppl 1):23-5.

6. Tang ST, Huang E-W, Liu T-W, Wang H-M, Rau K-M, Chen J-S, et al. Aggressive end-of-life care significantly influenced propensity for hospice enrollment within the last three days of life for Taiwanese cancer decedents. J Pain Symptom Manag. 2011;41(1):68-78.

7. https://www.cancer.gov/about-cancer/treatment/types/chemotherapy.

8. Zhang Z, Chen M-L, Gu X-L, Liu M-H, Zhao W-W, Cheng W-W, et al. Palliative chemotherapy near the end of life in oncology patients. Am J Hosp Palliat Med. 2018;35(9):1215-20.

9. Akin S, Can G, Aydiner A, Ozdilli K, Durna Z. Quality of life, symptom experience and distress of lung cancer patients undergoing chemotherapy. Eur J Oncol Nurs. 2010;14(5):400-9.

10. Harrington SE, Smith TJ. The role of chemotherapy at the end of life: "when is enough, enough?" JAMA. 2008;299(22):2667-78.

11. Lewin SN, Buttin BM, Powell MA, Gibb RK, Rader JS, Mutch DG, et al. Resource utilization for ovarian cancer patients at the end of life: how much is too much? Gynecol Oncol. 2005;99(2):261-6.

12. Temel J, Greer J, Gallagher E, Admane S, Pirl W, Jackson V, et al. Effect of early palliative care (PC) on quality of life (QOL), aggressive care at the end-of-life (EOL), and survival in stage IV NSCLC patients: results of a phase III randomized trial. J Clin Oncol. 2010;28(15 suppl):7509.

13. Pacetti P, Paganini G, Orlandi M, Mambrini A, Pennucci MC, Del Freo $A$, et al. Chemotherapy in the last 30 days of life of advanced cancer patients. Support Care Cancer. 2015;23(11):3277-80.
14. Skov Benthien K, Adsersen M, Petersen MA, Soelberg Vadstrup E, Sjøgren $P$, Groenvold MJ. Is specialized palliative cancer care associated with use of antineoplastic treatment at the end of life? A population-based cohort study. Palliat Med. 2018;32(9):1509-17.

15. Chan WL, Lam KO, Siu WK, Yuen KK. Chemotherapy at end-of-life: an integration of oncology and palliative team. Support Care Cancer. 2016;24(3):1421-7.

16. Hui D, Didwaniya N, Vidal M, Shin SH, Chisholm G, Roquemore J, et al. Quality of end-of-life care in patients with hematologic malignancies: a retrospective cohort study. Cancer. 2014;120(10):1572-8.

17. Liu T-W, Chang W-C, Wang H-M, Chen J-S, Koong SL, Hsiao SC, et al. Use of chemotherapy at the end of life among Taiwanese cancer decedents, 2001-2006. Acta Oncol. 2012;51(4):505-11.

18. Sano M, Fushimi K. Association of palliative care consultation with reducing inpatient chemotherapy use in elderly patients with cancer in Japan: analysis using a nationwide administrative database. Am J Hosp Palliat Med. 2017;34(7):685-91.

19. Bossuyt PM, Reitsma JB, Bruns DE, Gatsonis CA, Glasziou PP, Irwig LM, et al. The STARD statement for reporting studies of diagnostic accuracy: explanation and elaboration. Ann Intern Med. 2003:138(1):W1-12.

20. de Oliveira MRF, de Castro Gomes A, Toscano CM. QUADAS and STARD: evaluating the quality of diagnostic accuracy studies. SciELO Public Health. 2011;45:416-22

21. Miles MB, Huberman AM, Saldaña J. Qualitative data analysis: a methods sourcebook. 3rd ed. Thousand Oaks, CA: Sage; 2014.

22. Bisht M, Bist S, Dhasmana D, Saini S. Effect of palliative drug therapy on quality of life in advanced head and neck cancer patients. Indian J Otolaryngol Head Neck Surg. 2011;63(3):220-2

23. Groene O, Crosby T, Hardwick RH, Riley S, Greenaway K, Cromwell D. A population-based observational study on the factors associated with the completion of palliative chemotherapy among patients with oesophagogastric cancer. BMJ Open. 2015;5(3):e006724.

24. Näppä U, Lindqvist $O$, Rasmussen $B$, Axelsson B. Palliative chemotherapy during the last month of life. Ann Oncol. 2011;22(11):2375-80.

25. Fujisawa D, Temel JS, Traeger L, Greer JA, Lennes IT, Mimura M, et al. Psychological factors at early stage of treatment as predictors of receiving chemotherapy at the end of life. Psycho-Oncology. 2015;24(12):1731-7.

26. Kao SC, van Zandwijk N, Corte P, Clarke C, Clarke S, Vardy J. Use of cancer therapy at the end of life in patients with malignant pleural mesothelioma. Support Care Cancer. 2013;21(7):1879-84.

27. Mayrbäurl B, Giesinger JM, Burgstaller S, Piringer G, Holzner B, Thaler J. Quality of life across chemotherapy lines in patients with advanced colorectal cancer: a prospective single-center observational study. Support Care Cancer. 2016;24(2):667-74.

28. Mayrbäurl B, Wintner LM, Giesinger JM, Himmelfreundpointner T, Burgstaller S, Holzner B, et al. Chemotherapy line-associated differences in quality of life in patients with advanced cancer. Support Care Cancer. 2012;20(10):2399-405.

29. Wintner LM, Giesinger JM, Zabernigg A, Sztankay M, Meraner V, Pall G, et al. Quality of life during chemotherapy in lung cancer patients: results across different treatment lines. Br J Cancer. 2013;109(9):2301-8.

30. Hyun MK, Jung KH, Yun YH, Kim YA, Lee WJ, Do YR, et al. Factors associated with place of death in Korean patients with terminal cancer. Asian Pac J Cancer Prev APJCP. 2013;14(12):7309-14.

31. Lehto RH, Miller SE, Flanigan M, Wyatt G. Mental health in patients with advanced cancer at the end of life: evaluation of evidence and future directions. Expert Rev Qual Life Cancer Care. 2018;3(23):73-94.

\section{Publisher's Note}

Springer Nature remains neutral with regard to jurisdictional claims in published maps and institutional affiliations. 